\title{
UCRL-PROC-213375
}

LAWRENCE LIVERMORE N A T IO N A L LABORATORY

\section{Construction and testing of wavefront reference sources for interferometry of ultra-precise imaging systems}

M. A. Johnson, D. W. Phillion, G. E. Sommargren, T. A. Decker, J. S. Taylor, Y. Gomei, O. Kakuchi, S. Takeuchi

July 1,2005

SPIE Optical Manufacturing and Testing VI

San Diego, CA, United States

July 31, 2005 through August 4, 2005 
This document was prepared as an account of work sponsored by an agency of the United States Government. Neither the United States Government nor the University of California nor any of their employees, makes any warranty, express or implied, or assumes any legal liability or responsibility for the accuracy, completeness, or usefulness of any information, apparatus, product, or process disclosed, or represents that its use would not infringe privately owned rights. Reference herein to any specific commercial product, process, or service by trade name, trademark, manufacturer, or otherwise, does not necessarily constitute or imply its endorsement, recommendation, or favoring by the United States Government or the University of California. The views and opinions of authors expressed herein do not necessarily state or reflect those of the United States Government or the University of California, and shall not be used for advertising or product endorsement purposes. 


\title{
Construction and testing of wavefront reference sources for interferometry of ultra-precise imaging systems
}

\author{
Michael A. Johnson ${ }^{* a}$, Donald W. Phillion ${ }^{\mathrm{a}}$, Gary E. Sommargren ${ }^{\mathrm{a}}$, Todd A. Decker ${ }^{\mathrm{a}}$, \\ John S. Taylor ${ }^{\mathrm{a}}$, Yoshio Gomei ${ }^{\mathrm{b}}$, Osamu Kakuchi ${ }^{\mathrm{b}}$, Seiji Takeuchi ${ }^{\mathrm{b}}$ \\ ${ }^{a}$ Lawrence Livermore National Laboratory, P.O. Box 808, Livermore, CA, USA 94550-0808 \\ ${ }^{\mathrm{b}}$ Canon Inc., 23-10 Kiyohara-Kogyo-Danchi, Utsunomiya-Shi, \\ Tochigi 321-3298, Japan
}

\begin{abstract}
We have built and calibrated a set of 532-nm wavelength wavefront reference sources that fill a numerical aperture of 0.3 . Early data show that they have a measured departure from sphericity of less than $0.2 \mathrm{~nm}$ RMS (0.4 milliwaves) and a reproducibility of better than $0.05 \mathrm{~nm}$ rms. These devices are compact, portable, fiber-fed, and are intended as sources of measurement and reference waves in wavefront measuring interferometers used for metrology of EUVL optical elements and systems. Keys to wave front accuracy include fabrication of an $800-\mathrm{nm}$ pinhole in a smooth reflecting surface as well as a calibration procedure capable of measuring axisymmetric and non-axisymmetric errors.
\end{abstract}

Keywords: interferometer, EUVL, wavefront measurement, phase-shifting interferometry

\section{INTRODUCTION}

Early in the development of extreme ultraviolet lithography (EUVL) it was recognized that fabrication of normalincidence diffraction-limited mirrors capable of operating at a $13-\mathrm{nm}$ wavelength was one of the key risk areas. It was deemed impractical to use EUV-wavelength metrology during the polishing cycle of these mirrors - first, because installing a soft $\mathrm{x}$-ray wavefront measuring interferometer at a polishing facility seemed very difficult and, second, the low thermal expansion materials chosen for mirror substrates had insignificant reflectivity at EUV wavelengths, thus requiring temporary coatings to be applied between polishing steps. In response, we developed the phase-shifting diffraction interferometer (PSDI) ${ }^{1}$, which operates at $532 \mathrm{~nm}$ and was used to measure mirror surface errors during fabrication of mirrors for the diffraction-limited wide-field EUVL cameras for the Engineering Test Stand ${ }^{2}$, as well as to test the transmitted wavefront during alignment. Verified later by EUV interferometry, this first-generation PSDI demonstrated wavefront measurement with a precision of $0.1 \mathrm{~nm}$ RMS and an accuracy of $0.5 \mathrm{~nm}$ RMS over a numerical aperture (NA) of 0.1 .

We report here the construction and calibration of the key element of a second-generation PSDI-a 532-nm wavefront reference source that fills a 0.3 NA and whose wavefront has a measured departure from sphericity of less than $0.2 \mathrm{~nm}$ RMS ( 0.4 milliwaves) and a reproducibility of better than $0.05 \mathrm{~nm}$ RMS. In addition to substantial hardware improvements, we have developed novel calibration procedures and propagation algorithms allowing measurement of both rotationally symmetric and non-symmetric wavefront errors. While the principal use of this technology will continue to be for metrology of EUVL components and systems, we see wide applicability for metrology of other ultra-precise optical systems, such as X-ray imaging optics for laboratory and astronomical instruments, as well as for large-aperture visiblewavelength telescopes.

\section{REQUIREMENTS}

\subsection{Application to EUVL}

Figure 1 shows a typical application for the new wavefront reference source (WRS). One WRS is placed at a field point of an EUVL projection optics system and sends a measurement wave through the optical system. A second WRS at the conjugate image point produces a reference wave from a small pinhole and also reflects the measurement wave at a 
point near the pinhole. Both waves propagate to a CCD chip where multiple interferences pattern are recorded. Analysis produces a transmitted wavefront referenced to the system pupil. Our goal for accuracy of such a PSDI system is 0.25 $\mathrm{nm}$ RMS with a reproducibility of less than $0.1 \mathrm{~nm}$ RMS. These specifications are to apply to the total error contained in the first 36 Zernike modes.

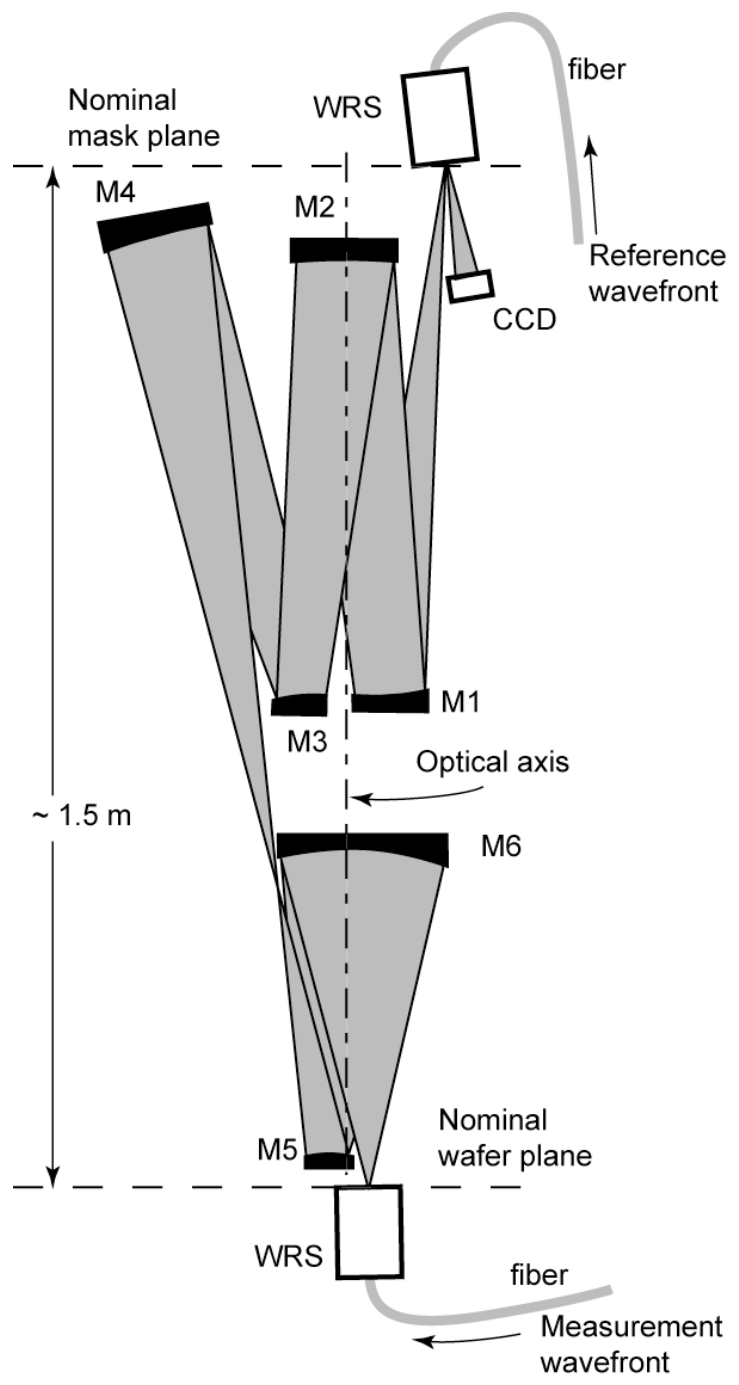

Figure 1. Schematic of potential 6-mirror EUVL camera showing use of wavefront reference sources to measure transmitted wavefront. Lower WRS supplies measurement wave; upper supplies reference wave and reflects measurement wave. CCD camera records interference pattern between the two waves. The illustrated optical system was designed by R. Hudyma (LLNL), US Patent 6,033,079.

\subsection{Two key requirements}

There are two key requirements on the WRS - (1) quality and stability of the emitted measurement and test wavefronts and (2) quality of the reflecting surface near the reference pinhole. Overall simplicity of the design-note the lack of beam splitters, turning mirrors or camera imaging optics - is what places the burden on these two requirements.

The basic design of the WRS therefore involves the illumination of a small pinhole etched into a reflecting mirror. The size and quality of the pinhole determines the numerical aperture and sphericity of the emitted beam. The flatness of the mirror at certain critical spatial frequencies determines the fidelity of the reflected measurement wave. 


\subsection{Other requirements}

In addition to these two main requirements on the WRS, we wanted to incorporate several other features. First we wanted a portable WRS, one that could be aligned and calibrated before it was installed in the EUVL system to be measured. This portability would also allow interchangeability and redundancy, thus allowing cross calibration. Therefore, we supplied the WRS with light via optical fiber. This portability also required a reproducible mechanical interface to the main PSDI structure. For this WRS interface we designed kinematic supports of the minimal restraint type.

Control of optical polarization was a further requirement. Electromagnetic (EM) field calculations of propagation through a small pinhole showed us that differences in wavefront sphericity between linear and circular polarization could be expected at the $0.1 \mathrm{~nm}$ level. Furthermore, we anticipated that the large angles of incidence occurring in highNA EUVL systems could cause polarization dependent phase shifts. We therefore incorporated diagnostic polarization analyzers into the WRS design so that specific polarization states could be produced. For our measurements with the axially symmetric test lens we took advantage of the symmetry of circular polarization passing through a circular pinhole whenever possible.

Next, we calculated effects on the emitted wavefront of possible misalignment of the internal optics of the WRS. We found that moveable internal alignment masks were needed to adequately center the illumination spot on the back of the pinhole. We also employed numerical simulations to determine the attenuation of aberrations by the pinhole. These calculations $^{3}$ showed that the wavefront arriving at the pinhole would have to have less than 0.25 waves of coma and less than 0.15 waves of astigmatism in order to meet the goal for sphericity of the transmitted wavefront. Higher order aberrations are more strongly filtered by the pinhole and have correspondingly looser specifications.

Finally, our calibration algorithms required that the WRS assembly be rotated precisely around its own optical axis as well as around an oblique axis passing through the pinhole. This mechanical motion, as well as movement of the polarization analyzers and the alignment masks, would all have to take place in vacuum via remote control. In this regard, our consideration of non-uniform thermal loading in vacuum led us to require that all motors and sensors draw zero current during a wavefront measurement.

\section{HARDWARE CONFIGURATION}

Figure 2 shows the key features of the final hardware design of the WRS. Laser light is supplied by a single-mode optical fiber. The fiber tip is aligned to the optical axis by a piezo-driven two-axis stage (range of motion $= \pm 40 \mu \mathrm{m}$ ). Light from the fiber is then collimated and passed through one of seven apertures in a motor-driven wheel. Three of the openings contain alignment masks and four of the openings contain polarization analyzers - two linear and two circular. Light from the analyzers is diverted by small prisms to a photodiode. We use these diagnostics only during setup procedures; the light passes through a clear aperture during wavefront measurement.

Next the light is focused by a lens mounted on a motor-driven axial flexure. This flexure permits precision focus control without allowing tilt or decentering. The combination of the collimating and focusing lenses produces a magnification of 0.5 at the pinhole with respect to the fiber tip. The measured aberrations in the wavefront arriving at the pinhole were $15 \mathrm{~nm}$ ( 0.03 waves) of coma, $7 \mathrm{~nm}(0.01$ waves) of astigmatism and $27 \mathrm{~nm}$ ( 0.05 waves) of primary spherical aberration, all of which meet the specifications.

\subsection{Pinhole mirror}

The pinhole mirror is the single most important component of the WRS. Our EM calculations showed that a pinhole diameter of $800 \mathrm{~nm}$ or less is required in order to fill a numerical aperture of 0.3 with less than 2:1 intensity variation from center to edge. Furthermore, the pinhole perimeter must have less than $4 \mathrm{~nm}$ RMS variation from a true circle. Presence of the low-order odd mode numbers ( 3 and 5 cycles on the circumference) produces the most significant wavefront departure from sphericity; the even-numbered modes are much less important.

Another requirement on the pinhole is that the opaque substrate region around the opening must have a transmission no greater than $10^{-13}$ in order to prevent leaked light from perturbing the phase of the main transmitted wavefront. In practice, this high opacity restricts the materials in which the pinhole can be cut to metals that have sufficient optical at- 
tenuation at a thickness less than the diameter of the pinhole. It would be difficult to drill an accurate pinhole in much thicker substrates and the resulting "tunnel" could have unacceptably low transmission.

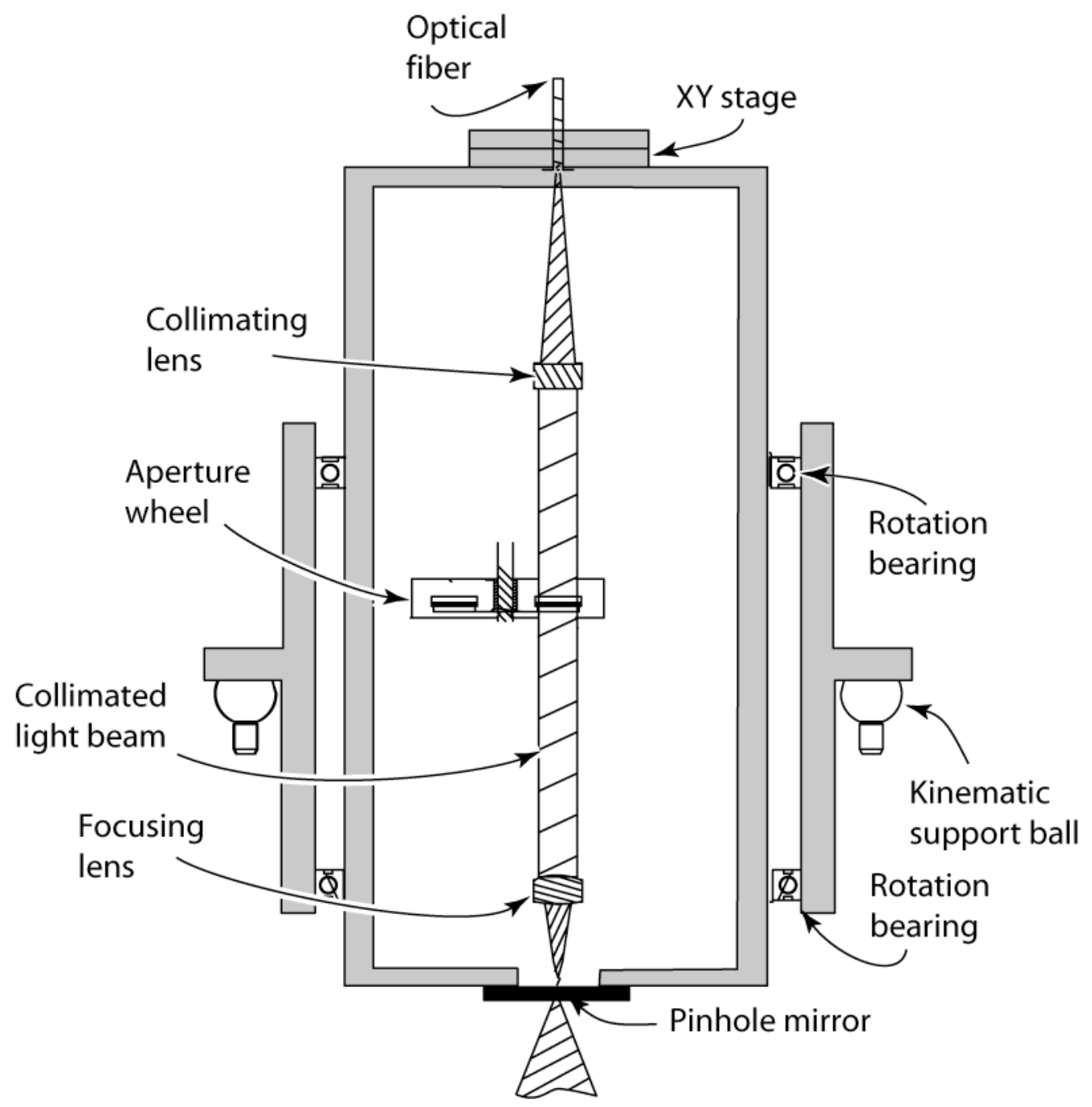

Figure 2. Schematic cross section of wavefront reference source (WRS). Overall height is about $20 \mathrm{~cm}$; weight is about $4 \mathrm{~kg}$; material is stainless steel. Optical fiber at top supplies 532-nm light to collimating lens. Light beam passes through aperture wheel to focusing lens, which focuses light on back of pinhole mirror. Kinematic support balls fix WRS to external platform. Bearings allow rotation of internal WRS structure around optical axis. Motors for aperture wheel, WRS rotation, and focusing, electronic components for polarization analysis, and electrical interfaces are not shown.

The last requirement on the pinhole mirror is its flatness; the reflected measurement wave must not have significant errors introduced by the mirror. As a relevant example, we knew that we could produce ${ }^{2}$ EUVL optics with multilayer coatings that had a mid-frequency roughness ( $1 \mu \mathrm{m}$ to $1 \mathrm{~mm}$ period) of $0.3 \mathrm{~nm}$ RMS. Our numerical simulations of reflections from such surfaces showed that similar flatness of the pinhole mirror would be adequate.

Based on these requirements for the pinhole mirror we settled on the following design:

1) We began with a super-polished single-crystal $200-\mathrm{mm}$ diameter Si wafer that was $0.5 \mathrm{~mm}$ thick. Thirty two individual $12-\mathrm{mm}$ square substrates were laid out on the wafer. 
2) We coated the wafer with silicon nitride, masked on the back with photo resist and etched with $\mathrm{KOH}$ to produce free-standing silicon nitride windows that were $0.2 \mu \mathrm{m}$ thick and $40 \mu \mathrm{m}$ square.

3) To achieve a high optical opacity, the silicon nitride was coated with a $0.5-\mu \mathrm{m}$ thick Mo/Si multilayer (76 pairs). The ratio $2.5: 1$ of Mo to Si thicknesses was adjusted to produce a low tensile stress (40 MPa) in the free-standing membrane, ensuring its flatness ${ }^{4}$.

4) The 700-nm diameter pinhole was drilled with a focused ion beam etching (FIBE) tool using a spot size of approximately $30 \mathrm{~nm}$.

5) After drilling, we diced the Si wafer into 12-mm square pinhole mirrors.

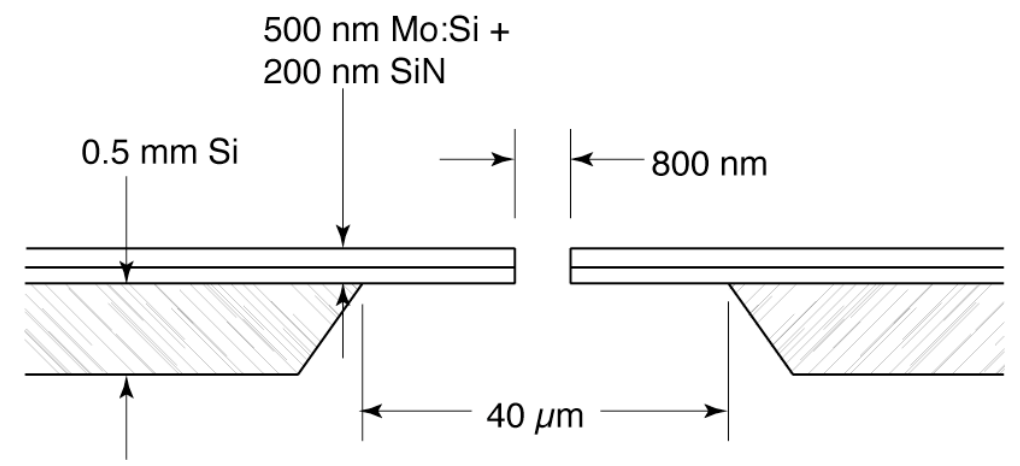

Figure 3. Schematic cross section of pinhole mirror. Substrate is 0.5-mm thick single crystal silicon. First layer is chemically produced silicon nitride; top layer is sputtered Mo/Si multilayer. Pinhole is etched by FIBE.

Figure 3 shows a schematic cross section of one pinhole mirror. After several trials to adjust the stress in the Mo/Si multilayer, we found the pinhole production process to be straightforward. The overall yield of about $30 \%$ was limited principally by blemishes in the silicon nitride coating. Figure 4 is an SEM picture of a typical pinhole. In all cases the pinholes were smooth and very round, with typical departures from circular of $1.2 \mathrm{~nm}$ RMS.

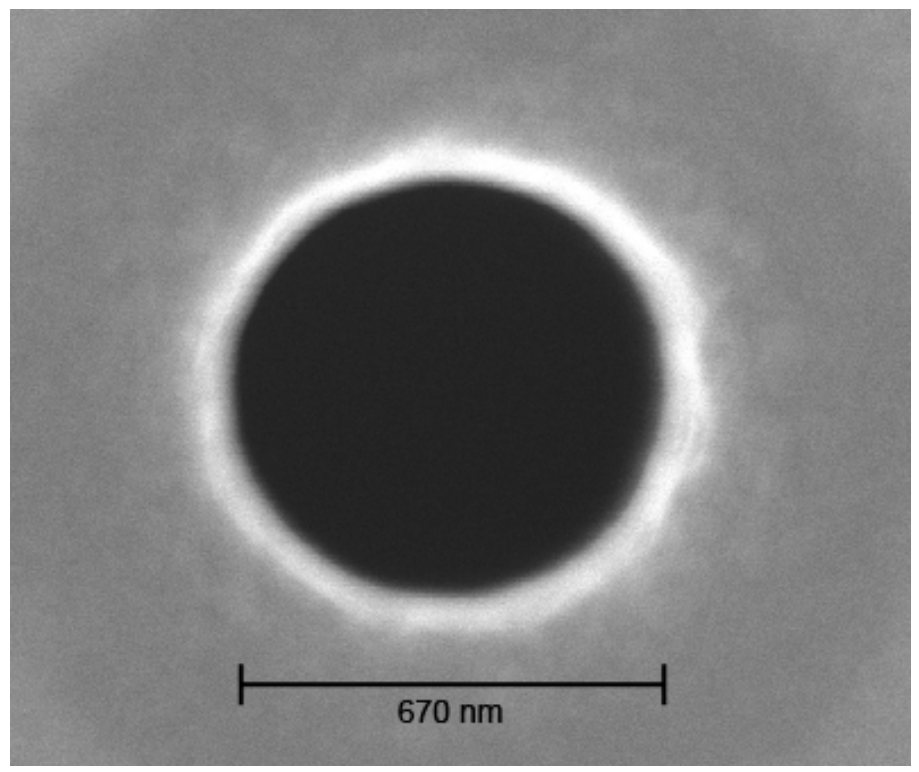

Figure 4. Scanning electron microscope picture of FIBE-etched pinhole. This pinhole was installed in one of the four wavefront reference sources. Its perimeter departs from circular by $1.2 \mathrm{~nm}$ RMS. 
We measured the flatness of the pinhole mirrors with a white-light interferometer. Figure 5 shows a typical surface height map. We have superimposed the approximate location of the silicon nitride window on this map. We also show the locations we used for the $6-\mu \mathrm{m}$ diameter reflected measurement beam.

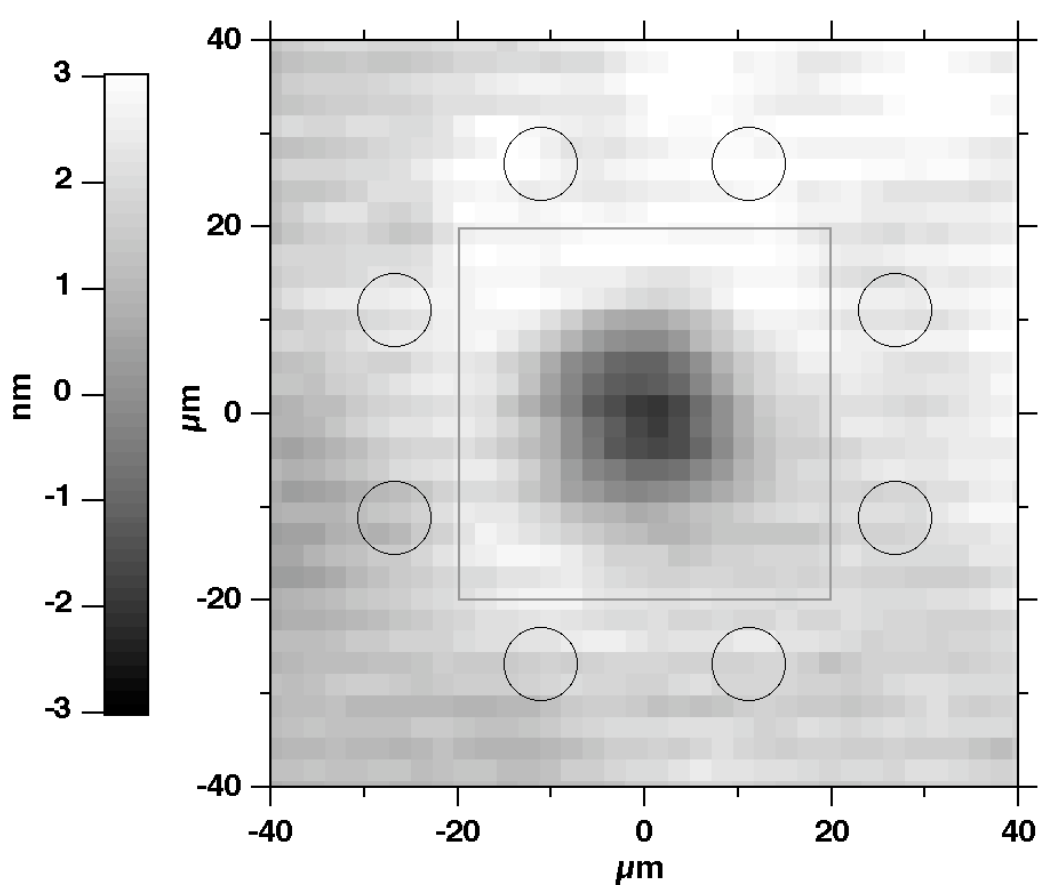

Figure 5. Surface height map of area around hole in pinhole mirror. (Same pinhole shown in Fig. 4).

Gray square is approximate location of silicon nitride window. Gray circles show approximate size and 8 locations of reflected 6- $\mu \mathrm{m}$ diameter measurement beam. Square $2-\mu \mathrm{m}$ pixels in image show resolution of measurement.

\subsection{Mechanical construction}

The primary considerations in the overall mechanical design of the WRS were stability and reproducibility. Anticipating operation in vacuum on precision stages during calibration of an EUVL stepper, we also analyzed thermal signature, mass moments, and vibrational modes. We minimized thermal loading by ensuring that no motor current would be drawn during a measurement cycle and that the least possible current be drawn for movements required between measurements. The weight of a WRS was about $4 \mathrm{~kg}$, with the center of gravity located in the plane of the kinematic supports. After assembly we tested the vibrational response on a shake table and verified that the lowest mechanical resonance occurred at about $400 \mathrm{~Hz}$. During optical testing we also verified that run out on the rotation bearings was less than $2 \mu \mathrm{m}$. We manufactured a total of four identical WRS units.

In order to calibrate the four wavefront reference sources we assembled them in pairs as shown in Fig. 1. However, instead of an EUVL 6-mirror camera we constructed a special lens as a test vehicle (lens provided by Canon). This lens had a magnification of 4:1 and a numerical aperture on the object side of 0.3 . We also mounted each WRS on a flexuresupported 5-axis stage that allowed us to move each WRS a few millimeters in any direction. Finally, we mounted these stages, the test lens, and the CCD camera on a stable truss inside a vacuum chamber, which we evacuated to a pressure of a few tenths of a torr. 


\section{CALIBRATION AND TEST PROCEDURES}

\subsection{Alignment}

After assembling the system, we aligned it so that the two pinholes lay on the rotation axis of the test lens as well as at optically conjugate axial positions that minimized aberrations in the transmitted wavefront. We accomplished this alignment by using a combination of an auxiliary camera and alignment laser as well as by iterating the WRS positions while measuring the transmitted wavefront as described below.

\subsection{Test procedure}

We employed a 4-step test procedure in order to separate the wavefront errors of each WRS from the much larger errors in the test lens. We measured the transmitted wavefront of the assembled test system for each of the following configurations:

1) Four rotational positions of the lower WRS - where the rotations were made about the optical axis of the WRS. This allowed determination of the non-axisymmetric wavefront errors of the lower WRS.

2) A further 4 rotational positions of the lower WRS - where the rotations were made about an oblique axis passing through the pinhole at an angle of $6.9^{\circ}$ to the optical axis of the WRS. This allowed determination of the axisymmetric wavefront errors of the lower WRS.

3) Interchange of the upper and lower WRS's followed by repetition of steps (1) and (2).

4) Rotation of the test lens about its optical axis while holding the WRS's fixed. This test served as a redundancy check on steps (1)-(3).

\subsubsection{Averaging process}

We began by adjusting the position of the lower WRS so that the image location of its pinhole on the upper WRS pinhole mirror fell at one of the eight locations shown in Fig. 5. We could accurately find this location by observing the spatial frequency and orientation of the fringe pattern on the CCD camera.

For each configuration of the two wavefront references and the test lens, the measurement of the transmitted wavefront consisted of a nested set of averages. The most basic element of these averages was the capture of a single phase/amplitude map. To get this map we ramped the phase of the measurement wave in 12 steps of $45^{\circ}$ phase, taking $30 \mathrm{~ms}$ for each step. At each step we recorded an interferogram consisting of a $1 \mathrm{~K}$ by $1 \mathrm{~K}$ frame of 12-bit pixels. We used a 12-bucket algorithm ${ }^{5}$ to reduce these 12 frames to the measurement wave's amplitude and phase present at the plane of the CCD. Typically, we immediately repeated this process 8 times, averaging the complex phasors to form a single phase/amplitude map, which we stored. We than translated the lower WRS in order to move the reflected spot on the upper pinhole mirror to the next of the 8 locations and repeated the capture of 8 averaged phase/amplitude maps. Because of the offset at the pinhole mirror between the spot from the measurement wave and the pinhole for the reference wave, the two waves arriving at the CCD had a relative tilt and produced a set of more-or-less straight fringes. We refer to this set of 8 positions around the reference pinhole as a "tilt grid."

\subsubsection{Tilt grid reduces potential systematic and random errors}

There are a number of reasons to use the tilt grid. First and most obvious, the spot from the measurement wave does not strike the pinhole. Although this spot is at least four times the diameter of the pinhole, there is no reason to allow the potential distortion of such an overlap. Furthermore, we use a tilt grid with inversion symmetry with respect to the pinhole in order to cancel to first order the systematic distortion that is introduced into the wavefront when a single tilted interferogram is propagated back to the system pupil. Finally, averaging over many points in the tilt grid reduces the random effects of pinhole mirror roughness and of the possible print through of the fringe modulation onto the wavefront phase. In particular, using a relatively large tilt grid means that the print through from the correspondingly high fringe frequency cannot affect the low order wavefront errors.

\subsubsection{Numerical propagation to pupil}

The results from a set of data taken on a tilt grid are 8 phase/amplitude maps localized at the plane of the CCD. There are no optical elements imaging the plane of the CCD to pupil of the test lens; we do this imaging numerically. We use standard methods ${ }^{6}$ of $\mathrm{ABCD}$ ray-matrix optics and paraxial propagation based on the scalar wave equation to propagate 
each interferogram from the CCD to the system pupil. At that point we average the 8 pupil wavefronts from a tilt grid and remove the mean piston, tilt, and focus to form the final result for the transmitted wavefront.

\subsubsection{WRS rotations and exchanges}

Next we perform the four steps outlined in Sec. 4.2, rotating the lower WRS and the test lens in order to separate the relatively large wavefront errors (about $10 \mathrm{~nm}$ RMS) in the test lens from the much smaller errors in the lower WRS wavefront. Finally, we exchanged all 4 WRS's in the test system in various combinations.

\subsection{Redundancy and data reduction}

The mathematics of the analysis method used for these data are described in a companion paper ${ }^{7}$. In summary, the WRS emitting the high NA measurement wave is rotated about its optical axis to four positions separated by $90^{\circ}$ with respect to the combined test lens and reference WRS. Simple subtraction of the transmitted wavefronts produces all Zernike coefficients for the measurement WRS except those that have 4-fold axial symmetry. Rotations of the measurementwave WRS about an oblique axis break the symmetry of these axially symmetric errors and again allow their separation from errors in the combined test lens and reference-wave WRS. We show in the above reference that four axial and four oblique rotations of the measurement WRS allows us to determine the first 16 Zernike coefficients of the wavefront from the measurement WRS. In practice, we ignore the coefficients for piston, tilt, and focus because these modes are irrelevant to the calibration.

In principal, it is possible with $N$ axial and $N$ oblique rotations to determine the first $N^{2}$ Zernike coefficients. However, we were not surprised to find that virtually all of the error in the measurement wave consisted of astigmatism, the lowest order aberration. Our analysis of the pinhole roundness, the quality of the focusing optics and the spatial filtering ability of the pinhole had led us to predict this conclusion early in the design process.

\section{DATA AND ANALYSIS}

\subsection{WRS wavefront sphericity}

Following the procedure outlined above, we are making measurements of the departure from sphericity of the wavefronts emitted by each of the four wavefront reference sources. Figure 6 shows an early wavefront map of a WRS, plotted as the departure from sphericity. Under the best operating conditions we have been able to achieve a departure from sphericity of less than $0.2 \mathrm{~nm}$ RMS for one of the four WRS's. A second WRS has a departure of $0.5 \mathrm{~nm}$ RMS. The third and fourth WRS are presently being measured. In all cases the dominant mode of departure is astigmatism.

\subsection{Transmitted wavefront reproducibility}

Of course, the ultimate use we see for a set of calibrated WRS's is the measurement of the transmitted wavefront of an ultra-precise optical system, such as an EUVL camera. The WRS wavefronts will contain only known low-order aberrations (which can be accounted for), but the wavefront of the optical system under test can be measured out to much higher order aberrations, limited only by the random errors that occur during that measurement. Therefore, we are treating the test lens as such an optical system and make checks of short-term and long-term reproducibility of the system's transmitted wavefront by varying the timing and other conditions of the WRS substitutions. We typically included the first 36 Zernike coefficients in these data.

Table 1 summarizes the numerical results of the first series of these tests. All of the data in Table 1 are the results from averaging phase/amplitude maps over a full tilt grid, as described in Sec. 4.2. All include the first 36 Zernike coefficients. Statistics are based on five system wavefronts for each WRS. We calculated the RMS deviation of each wavefront from the mean wavefront of the group and report the average of the five RMS values.

\begin{tabular}{l|l|l|l|l} 
& WRS \#1 & WRS \#2 & WRS \#3 & WRS \#4 \\
\hline Short-term variation & $0.043 \mathrm{~nm}$ & $0.053 \mathrm{~nm}$ & $0.029 \mathrm{~nm}$ & under test
\end{tabular}

Table 1. RMS departures in $\mathrm{nm}$ from mean transmitted wavefront (set of 5). Total duration taken for all 5 wavefront measurements for one WRS is about 10 minutes. Variations include the first 36 Zernike coefficients. 


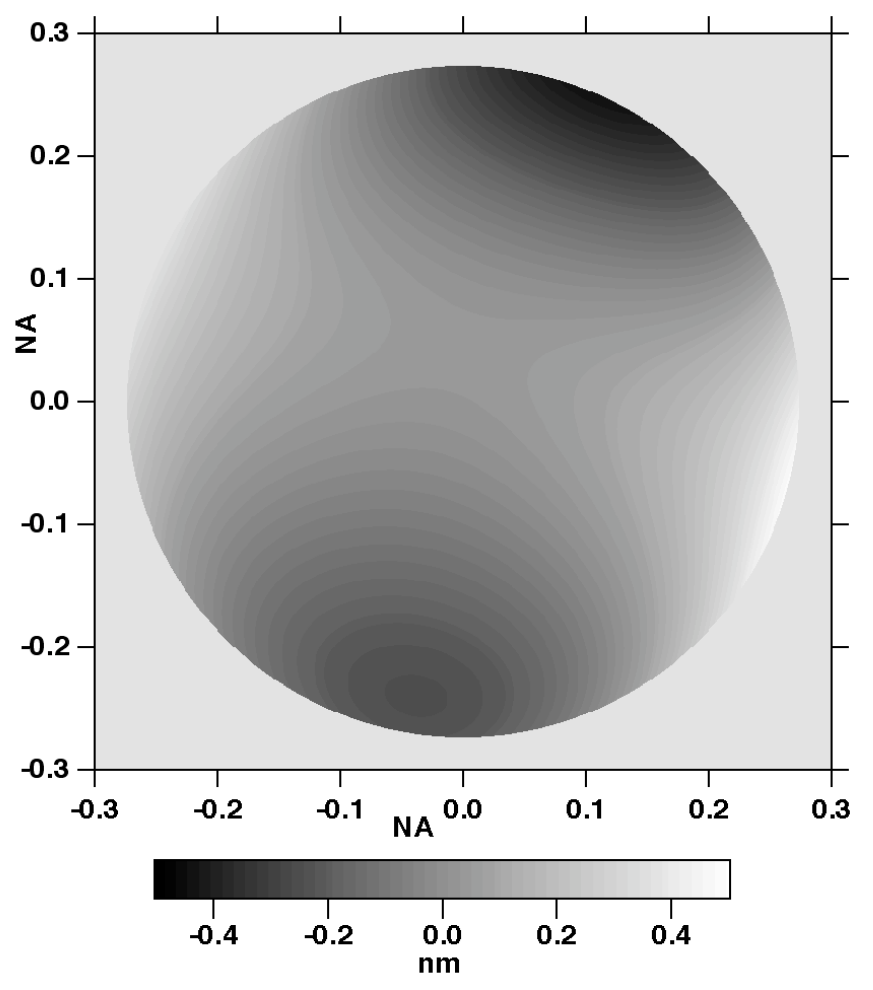

Figure 6. Measured departure from sphericity of WRS wavefront. Contributions from the first 16 Zernike polynomials are shown. RMS variation is $0.16 \mathrm{~nm}$; peak-to-valley is $0.82 \mathrm{~nm}$.

\section{CONCLUSIONS}

These early results show that our new wavefront reference sources can be aligned to have wavefronts that depart from sphericity by $0.2 \mathrm{~nm}$ RMS or less and have short-term stability of $0.05 \mathrm{~nm}$ RMS or less. Our ongoing measurement program has three phases studying stability. The first phase is study of stability over periods of a few minutes to about an hour during which we make no changes to the configuration. We will identify potential disturbances to the system, such as vibration and thermal transients. The second phase will study long-term stability - again without changes in configuration-where we consider diurnal disturbances. In the final test phase we will demonstrate the full potential of the WRS; we will show that the WRS is stable when removed completely from the vacuum system and later replaced. This, or course, is the mode in which a WRS will be calibrated in a test stand and then used in an EUVL projection optics system.

Our most important conclusion is that this technology substantially lowers the risk of producing projection optics for EUV lithography. Furthermore, this accuracy and precision come in large part from the simplicity in design and operability of these sources - two calibrated wavefront reference sources and a CCD array are the only components needed to measure the transmitted wavefront of an EUVL system; therefore the cost of such metrology can be a very small fraction of the system cost.

We see a natural extension from metrology of EUVL systems to laboratory soft X-ray cameras using compact normal incidence optics, to high-energy X-ray grazing incidence optics, as well as to astronomical X-ray telescopes. There is also evidently a need for metrology of ultra-precise visible-wavelength optics for space-based missions such as the terrestrial planet finder. The robust sub-nanometer metrology we have demonstrated could make contributions over this wide range of applications. 


\section{ACKNOWLEDGMENTS}

We gratefully acknowledge Lloyd S. Bradsher for assembly and operation of the PSDI, Terry D. Swan for mechanical design, Carl A. Chung for precision mechanical assembly, Sasa Bajt and Jennifer Alameda for pinhole substrate fabrication, Nhan Nguyen for CCD camera engineering, and Sherry Baker for profilometry.

This work was performed under the auspices of the U. S. Department of Energy by the University of California Lawrence Livermore Laboratory under Contract No. W-7405-ENG-48. Funding was provided by Canon Inc.

\section{REFERENCES}

1. G. E. Sommargren, et. al., "100-picometer interferometry for EUVL," Proceeding of SPIE, (4688) 316 (2002).

2. D. A. Tichenor, et.al., "System Integration and Performance of the EUV Engineering Test Stand," Emerging Lithographic Technologies V, Proceeding of SPIE (4343) 19 (2001).

3. K. A. Goldberg, "Extreme ultraviolet interferometry," Ph.D. Dissertation, University of California, Berkeley, 1997.

4. P. Mirkarimi, "Stress, reflectance, and temporal stability of sputter-deposited Mo/Si and Mo/Be multilayer films for extreme ultraviolet lithography," Opt. Eng. 38(7) 1246 (1999).

5. D. W. Phillion, "General methods for generating phase-shifting interferometry algorithms," Appl. Opt. 36(31) 8098 (1997).

6. A. E. Siegman, Lasers, Chapter 20, University Science Books, Mill Valley CA, 1986.

7. D. W. Phillion, et. al., "Calibration of symmetric and non-symmetric errors for interferometry of ultra-precise imaging systems," Proceeding of SPIE, August meeting, 2005. 